\title{
Optimization Method of Estimating the Leaf Mass of a Tree
}

\section{Zhongcheng Zhang}

\author{
School of science, Wuhan Institute of Technology, Wuhan 430073, Hubei, China \\ zzcheng63@126.com
}

Keywords: Leaf shape; Leaf mass; Simulation model

\begin{abstract}
Leaves have the various shapes, but there was no excellent method to estimate the actual weight of the leaves of a tree until now. Based on the methods of qualitative analysis, data fitting, and theoretical derivation, this paper establishes a simulation model on leaf mass and leaf shapes, then estimates the weight of the leaves. Then an example of estimating the leaves mass of a Birch tree is given to show the validity and feasibility of our model.
\end{abstract}

\section{Introduction}

These years, research about trees and trees on the ecological environment, and social activities, and other aspects of the influence of the research hasn't been stopped.

Mary Byrne, Marja Timmermans, Catherine Kidner and Rob Martienssen discussed about development of leaf shape in 2001[1]. Their findings highlight the importance of interactions between the shoot apical meristem (SAM) and developing leaf primordia in axis specification and the establishment of leaf shape. Silva Fenn researched Foliage and branch biomass estimation of coniferous and deciduous tree species in 2002[2], showed stem biomass is calculated from measurements of stem diameter and height by assuming a constant wood density and a certain shape of the trunk. Mencuccini[3] and Bonosi indicated stem diameter depend on environmental conditions such as drought stress in 2001. Claesson and Henskens[4] et al. used a number of investigations indicate a significant influence of stand density and individual competitive status on these relations. Bartelink and Mäkelä and Vanninen also suggested that the individual competition of a tree can be used for determining the relations between stem dimension and biomass in certain compartments.

According to these above found, further study are in progress, Mäkelä and Vanninen found the density of foliage biomass has been found to decrease with crown depth after a maximum that was observed approximately at the height of largest crown extension, therefore, the foliage biomass within the crown can be expected to be smaller in the shade because photosynthesis and thus carbon gain decreases with decreasing light availability. Thus, leaf area density at a particular crown location is directly or indirectly related to light availability at that point. This suggests that foliage biomass within a crown segment can be calculated from leaf area density and specific leaf area, if light availability can be estimated from other variables.

And the relationship between crown diameter and diameter at breast height (DBH) is obvious, and crown diameter is usually closely related to $\mathrm{DBH}$, so they are often used to calculate the stand growth, stand density and tree spacing. Duchaufour[5] was first to determine of the relationship between crown diameter and diameter at breast height. Subsequently, some scholars also determined the ratio of crown diameter and DBH for the conifers and broadleaved tree species in temperate and cold regions. Dawkins [6] drawn a conclusion that the range of DBH is 20 to $50 \mathrm{~cm}$ for a series of trees, and the function relationship between crown diameter and DBH can be approximated as a straight line. Many studies showed that there are closely relation between crown diameter and DBH. In 1989, $\mathrm{Wu}[7]$ studied the relationship between crown diameter and DBH by using the method of linear regression, and explored the method of determining the standard density. Dai[8] studied that the function relationship between crown diameter and $\mathrm{DBH}$ is a straight line. Here, we select some measured data of crown diameter and DBH on 10 kinds of broad-leaved trees given by [9], and explored the relationship between crown diameter and DBH. It will give important base to study the problem of estimating the leaf mass of a tree. 
On the basis of the found above, we related some competition factors to foliage density and specific leaf area, calculated foliage biomass from these two, and integrated the results over the whole crown. And we show a program to calculate the weight of tree leaves through the establishment of the simulation model.

\section{Models}

Tree can be divided into two parts, i.e., the trunk and crown. In order to study the impact factors of the leaf shape and estimate the actual weight of the leave, and crown dimension determines foliage biomass of a tree, we abstract a tree as a three-dimensional graph (see Figure 1). Moreover, the crown is divided into k segments by a given thickness dh (usually $0.5 \mathrm{~m}$ ). For each crown segment, a cone shaped space is constructed that is upside down with its apex at the centre of the segment. The angle $\beta_{1}$ is half of the apex of the competition cone (the angle between the vertical and the surface of the cone), which expands above the centre of each segment. A segment will be considered as a competition segment, if the angle $\beta_{\mathrm{x}}$, between the central and the competition segment is smaller than $\beta_{1}$. The angle $\beta_{1}=30^{\circ}$ is recommended by Pretzsch (1992) for whole crowns and has performed better than $10^{\circ}$ and $20^{\circ}$ openings that have been tested on the segment level. All volumes of competing segments are summed up into the overall competition value of the centre segment $\mathrm{CF}_{\mathrm{I}}$.

Additionally, half of the volume of the centre segment is also added to the competition value. Note that leaf area is determined from this factor and thus cannot be used as an independent variable to estimate radiation distribution

$$
C F_{I}=\sum_{1}^{c} V_{c}+V_{i} \times 0.5
$$

where $c$, number of segments within the competition cone; $V_{\mathrm{c}}$, volume of competing segment (in $\mathrm{m} 3$ ); $V_{\mathrm{i}}$, volume of centre segment $\left(\right.$ in $\left.\mathrm{m}^{3}\right)$.

LAD denotes the leaf area density of unit volume. The same quality leaves in the unit volume gathered the higher level, then the value of LAD is greater, which means the overlap is larger. On the contrary, LAD decreases, the overlap decreases.

$$
L A D=\frac{L A D_{\max }}{(C F+1)^{\text {plad }}}
$$

LADmax is maximum values. For a specific tree, a specific value was measured. plad, the sensitivity of the calculations, can set equal to $0.1,0.2,0.3$, and 0.4 . Above formula can be rewritten as

$$
\frac{L A D}{L A D_{\max }}=\frac{1}{(C F+1)^{\text {plad }}}
$$

We can conclude that relative lad is a decreasing function of the variable $\mathrm{CF}$, when $\alpha=1$, $\mathrm{LAD}=\mathrm{LAD}_{\max }$. Leaves gathered highest degree, mutual overlap is to be maximize, and CF close to 0 . At this point, competition index is minimum, volume of sun exposure is minimum. LAD decreased gradually, CF gradually increased, which indicates that the overlap between leaves is minimum so that volume of exposure in the sun is maximum. After CF is about 100, the curve gradually leveled off, which shows that when the LAD is to achieve an optimal value, LAD does not occur in significant changes, but the competition index continues to increase.

Specific leaf area denotes the sum of superficial area for per unit mass of leaves in the case of non -overlapping surface area. SLA can show the shape characteristics of the leaves.

$$
S L A=S L A_{\max }-\left(S L A_{\max }-S L A_{\min }\right) e^{[-p s l a(C F+1)]}
$$

where psla is scaling parameters, SLAmax is maximum specific leaf area. 
Independent variables of SLA and LAD are only CF, so these two curve can be plotted in the same coordinate axis, and then we can analyze the relationship between the two variable.

\section{(1) Compute the crown volume:}

Step 1: Compute the volume of each segment

Each segment can be regarded as a cylinder. The computation formula of cylinder surface area is $V=\pi r^{2} \times l$. Substitute the parameter given by us into $V=\pi r^{2} \times l$, the mean value of volume for each segment can be expressed as $V_{\mathrm{k}}=\pi \times(c d / 2)^{2} \times 0.5$.

Step 2: Compute the volume of all segments

Since stratification of crown is uniform, so the total crown volume is $V=V_{\mathrm{k}} \times k$.

Step 3: Calculate the volume of each segment

Each segment can be regarded as a cylinder. The computation formula of cylinder surface area is $V=\pi r^{2} \times l$. Substitute the parameter given by us into $V=\pi r^{2} \times l$, the mean value of volume for each segment can be expressed as $V_{\mathrm{k}}=\pi \times(c d / 2)^{2} \times 0.5$.

Since stratification of crown is uniform, so the total crown volume is $V=V_{\mathrm{k}} \times k$.

\section{(2) Calculate the crown radius}

\section{Step 1: Data collection}

The size, structure, shape of tree crown and its distribution in the stands directly determine the individual production, growth vigor and productivity, and reflect the growing conditions of trees in stands. Usually, the crown diameter is usually closely related to DBH. Many scholars did some valuable experiments to studies the between crown diameter and DBH.

In 2007, $\mathrm{Li}^{[9]}$ chose 10 kinds of representative broadleaved tree species, i.e., Fraxinus mandshurica, Phellodendron amurense, Juglans mand-hurica, Tilia Linn, Ulmus pumila, Acermono, Quercus mongolica, Betula platyphylla, Populus davidiana, Betula costata. Trees are positioned in the standard (determine the $\mathrm{x}$, y coordinates), and the diameter at breast height, tree height, the first living branch height and crown width and some other measurement factors were measured step by step.

Step 2: The relationship between crown diameter and DBH

Based on above data, we fit the function relationship between crown diameter and DBH by using the software of Matlab 7.0.

From above analysis, we can see that the function relationship between crown diameter and DBH can be approximated as a straight line, especially it is very obvious when the DBH is in the range between $10 \sim 30 \mathrm{~cm}$, and the crown diameter increased with the increase of DBH. In addition, the relationship between crown diameter and DBH of the other nine tree species can also show an obvious linear relationship. Here, we suppose that the fitting equations are as follows.

$$
c d=a+b D,
$$

where $c d$ is crown, $a$ is constant, $b$ is slope, $D$ is DBH.

Step 3 : Calculate the volume of space in the crown.

Considering the leaves distribution of trees, leaves have to do photosynthesis, that is, that leaves must expose to light. Therefore, we give an ideal model: Focus on all the leaves to the crown surface. But the side close to the trunk cannot accept enough light from the sun, then it will form a shadow that the thickness is the length of leaves, on the surface of the crown. According to the average crown diameter calculated in the previous step, the entire layered crown can be approximated as a cylinder, where the crown height is the cylinder height, the radius is the average crown diameter, and the volume of the leaves is the cylinder volume without bottom. The specific calculation process is as follows:

The volume of the internal space is

$V_{\text {int }}=\pi \times(c d / 2-2 \mathrm{~b})^{2} \times l$

where $l$ is the height of the cylinder. Then we get the total cylinder volume as follows:

$V_{\text {cyl }=} V-V_{\text {int }}$ 
Because a lot of leaves are oval-shaped, monolithic leaf area is calculated as follows:

$$
S={ }_{\pi} a b
$$

Because leaves are arranged in sequential order and single layer in the ring, so the outside radius of the circle is approximate as the half of the crown diameter, and the internal radius of the circle is the difference value between the half of the crown diameter and the length of foliage. Here, we suppose that the leaves arrange along the circle within the circular, then the amount of leaves arranged in circular is as follows:

$$
t=\frac{2 \times \pi \times\left(\frac{c d}{2}-2 b\right)}{2 a}
$$

Then the leaves area in the ircular is as follows:

$$
S_{\text {leaf }}=S \times t
$$

and the volume of all the leaves on the cylinder surface is

$$
\mathrm{V}_{\text {leaf }}=\mathrm{S}_{\text {leaf }} \times 1
$$

In the process of calculating the volume of leaves, we must consider that not only there are many leaves in the cylinder, but also the gap between the leaves. This gap will cause the leaves quality too large, and thus make the results inaccurate, so we should exclude the error caused by the gap, and it is necessary to use the foliage density f. Foliage density is defined as the leaf area divided by crown volume (units $\mathrm{m}^{-1}$ ).

From the definition of the foliage density $f$, we get the whole tree leaf area is

$$
S_{\text {all }}=V_{\text {leaf }} \times f
$$

The average thickness of the leaves is $m$, then the leaves volume that removed the gap is:

\section{(3) Calculate the leaves mass}

$$
V_{\text {all }}=S_{\text {all }} \times m
$$

The total mass of the leaves is as follows:

$$
\begin{gathered}
M=V_{\text {all }} \times \rho \\
=\pi a b \times \frac{2 \times \pi \times\left(\frac{a+b D}{2}-2 b\right)}{2 a} \times l \times \mathrm{m} \times \rho
\end{gathered}
$$

$$
=\frac{\pi^{2} b \operatorname{lm} \rho(a+b D-4 b)}{2}
$$

From this formula, we can conclude that leaves mass is closely related to the size of the features of the tree, more specifically,

(1) The height of the tree determined the height of the crown $l$.

(2) The height of the tree determined the tree's age and value D of DBH.

(3) The outline of the tree is closely related to the shape of the leaves, and the size of the tree determines the leaves mass.

\section{Conclusions}

In order to calculate the leaf mass of a tree, we translate this calculation problem into the problem of seeking the function relationship between crown diameter and DBH. By calculating the crown volume, the leaf mass is obtained. During the modeling process, we take some approximate treatments, for example, the crown is divided into many segments, and when calculating the crown volume, we approximate regard the crown as a cylindrical, this idealized model will inevitable cause a few errors. So the processing method needs to be further refined. 


\section{References}

[1] M. Byrne, Development of Leaf Shape, Current Opinion in Plant Biology 4 (2001) 38-43.

[2] R. Grote, Foliage and Branch Biomass Estimation of Coniferous and Deciduous Tree Species, Silva Fenn 36 (2002) 779-788.

[3] M. Mencuccini, L. Bonosi, Leaf/sapwood Area Ratios in Scots Pine Show Acclimation Across Europe, Can J For. 31 (2001) 442-456.

[4] F. L. Henskens, Battaglia M, Cherry ML, Beadle CL. Physiological basis of spacing effects on tree growth andform in Eucalyptus globules, Trees 15 (2001) 365-377.

[5] A. Duchaufour, L'ame'Agement de la Foret de Comp ie'gne, Revue Eaux et Foret 42 (1903) 65-68.

[6] H. C. Dawkins, Crown Diameter, Their Relation to Bole Diameter in Tropical Forest Trees, Commonwealth Forestry Rev. 42 (1963) 31-333.

[7] W. Z. De, Standard Density (Stem Number) to Determine the Method, Forest Inventory and Planning 2 (1989) 26-28.

[8] D. Fu, Research Crown Diameter and Diameter at Breast Height Relations of Ten Kinds of Major Natural Secondary Broad-leaved Tree in Maoershan, Bulletin of Botanical Research 29 (2009) 597-602. 